\section{DO FRACTURES CORRELATE WITH VITAMIN D LEVELS IN PRETERM INFANTS?}

M. McGann ${ }^{1}$, R. Mccarthy ${ }^{1}$, O. Uduma ${ }^{1}$, J. Brady², M. Mckenna ${ }^{2}$, B. Murray ${ }^{2}$, N. Murphy ${ }^{3}$, E. Molloy ${ }^{1,2,3,4}$

${ }^{1}$ Neonatology, National Maternity Hospital, ${ }^{2}$ Metabolism Laboratory, St Vincent's University Hospital, ${ }^{3}$ Edocrinology, Children's University Hospital, ${ }^{4}$ Royal College of Surgeons in Ireland, Dublin, Ireland

Background and Object: Vitamin D plays an important role in skeletal health. We previously reported a high prevalence (about $80 \%$ ) of serum 25-hydroxy-vitamin D (25OHD) insufficiency in preterm VLBW infants. We aimed to evaluate the incidence of inpatient fractures noted in this population and any association with vitamin D levels.

Methods: Convalescent, stable VLBW (birth weight $<=1.5 \mathrm{~kg}$ ) preterm (gestation $<=32$ weeks) infants, admitted to a tertiary referral NICU had serum 25-hydroxy-cholecalciferol (25-OH-D) measured using radio-immunoassay, prior to starting oral vitamin D supplements. All Xrays performed during inpatient stay were evaluated for the presence of fractures.

Results: 137 VLBW infants had 25-OH-D performed and all their Xrays reviewed. Their mean (+/-Standard deviation) gestation was 29.6+/-2.5 weeks, birth weight $1.3+/-0.4 \mathrm{~kg}$ and vitamin $D$ levels were $40.7+/-11.6 \mathrm{nmol} / \mathrm{l} .4(2.9 \%)$ infants had fractures : 3 had long bone fractures and 1 had rib fractures. Their mean vitamin D levels were $34.6+/-$ $10.9 \mathrm{nmol} / \mathrm{l}$.

Conclusions: We have demonstrated that $80 \%$ of preterm VLBW infants have insufficient 25-OH-D levels during early postnatal life; but $25 \%$ remain insufficient at follow-up assessment. Four infants had fractures and all had decreased Vitamin D levels $<50 \mathrm{nmol} / \mathrm{l}$.

\section{BODY COMPOSITION CHANGES IN EARLY POSTNATAL LIFE OF LATE PRETERM INFANTS}

\author{
P. Roggero, M.L. Giannì, N. Liotto, L. Morlacchi, \\ F. Mosca
}

NICU, Fondazione IRCCS Cà Granda - Ospedale Maggiore Policlinico,Department of Mother and Infant Sciences, University of Milan, Milan, Italy

Background and aims: Late preterm birth has recently increased. Several reports suggest that preterm infants have limited compensatory mechanisms to adapt to the extrauterine environment. Body composition may contribute to the "programming process". Aim of the present study was to assess the body composition changes through the first postnatal weeks in a cohort of late preterm infants.

Methods:Weconductedaprospective, observational study. Twenty-one infants ( 7 males) were included. Inclusion criteria: gestational age 34-36 weeks, singleton pregnancy, Caucasian parentage, born adequate for gestational age. Exclusion criteria: congenital/chromosomal, heart and brain diseases, necrotizing enterocolitis. Growth and body composition were assessed by an air displacement plethysmography system (PEA POD® Infant Body Composition System, LMI, USA) on the $5^{\text {th }}$ postnatal day of life and at term corrected age. Data on body composition (expressed as percentage of fat mass) were analyzed by the Friedman test.

Results: Mean gestational age (weeks) and birth weight $(\mathrm{g})$ were $35.5 \pm 0.68$ and $2665 \pm 239$, respectively. All infants were breast fed (human milk $>50 \%$ of the daily volume intake). Percentage of fat mass significantly increased from the 5th day $(7.15 \pm 3.76)$ of postnatal life to term corrected age $(16.1 \pm 4.3, p<0.001)$. Mean weight $(g)$ at term corrected age was $3428 \pm 352$.

Conclusions: Our data suggest that late preterm infants develop an increased adiposity as compared to full term infants. Further larger studies are required to investigate the long term health implication of this preliminary evidence. 